\title{
Measurement of Objective Video Quality in Social Cloud Based on Reference Metric
}

\author{
Sajida Karim (D), ${ }^{1}$ Hui He $\mathbb{D}^{1},{ }^{1}$ A. R. Junejo, ${ }^{2}$ and Mariyam Sattar ${ }^{3}$ \\ ${ }^{1}$ School of Computer Science and Technology, Harbin Institute of Technology, Harbin, China \\ ${ }^{2}$ School of Control Science and Control Engineering, Harbin Institute of Technology, Harbin, China \\ ${ }^{3}$ Department of Mechanical Engineering, Institute of Space Technology, Islamabad, Pakistan \\ Correspondence should be addressed to Hui He; hehui@hit.edu.cn
}

Received 20 June 2019; Revised 24 October 2019; Accepted 26 November 2019; Published 13 January 2020

Academic Editor: Jun Cai

Copyright (C) 2020 Sajida Karim et al. This is an open access article distributed under the Creative Commons Attribution License, which permits unrestricted use, distribution, and reproduction in any medium, provided the original work is properly cited.

\begin{abstract}
This paper explores the objective of the present video quality analysis (VQA) and measures the full reference metrics keeping in view the quality degradation. During the research work, we conduct experiments on different social clouds (SCs) and low-quality videos. Selected videos are uploaded to SC to assess differences in video service and quality. WeChat shows that the average of all videos (Avg = 100), peak signal-to-noise ratio (PSNR), has no impact on other indicators. Therefore, we believe that WeChat provides the best video quality and multimedia services to their users to meet Quality of Service (QoS)/Quality of Experience (QoE).
\end{abstract}

\section{Introduction}

At present, the number of social clouds (SCs) has exceeded the number of multimedia services, and the number of users joining the SC has also increased dramatically. This is because video access from the cloud such as video on demand (VOD), online video gaming, streaming multimedia, Internet protocol television (IPTV), video conferencing, and social networking has been widely used all over the world. These multimedia applications and services are now commonly implemented in various fields, including scientific and educational sectors (e.g., multimedia presentations) $[1,2]$.

Multimedia service providers (MSPs) devise various approaches and techniques to provide a better QoE that is diversely improved by the user experiences. The users can easily access the QoS from the commercial or noncommercial cloud servers. Therefore, the quality of multimedia services is more critical to the designs and deployed by any networks and services [3].

Users have always high expectations and demands for qualities of video. During the video transmission and online streaming, a single video may experience jitter or additional noise by uploading/downloading of videos on the cloud because the SC compresses the original video and reduces the storage size that automatically affects the video quality $[4,5]$.

The unnecessary noise increases the problem for MSP and reduces the provision of high definition (HD), highquality video services agreeing to the user demands. However, QoE and QoS are two modalities in multimedia societies and are highly interconnected to ensure the reliability of quality assessment (QA) that promises to provide QoS and improve the user QoE. Generally, QoE refers to the user factors, i.e., enjoyment, feelings, and satisfaction, while QoS refers to the performance of multimedia applications and networks, e.g., performance, responsiveness, and availability. Quality of Experience is further subcategorized into subjective and objective QoE. Subjective QoE is carried out by surveys (e.g., scale rate, interviews, and questionnaires). Objective QoE, on the other hand, carries out human physiological tests (e.g., MRI and EEG) and measures QoS data or technical parameters (e.g., cost, resolution, frame, and sampling rates) [4-6]. 
Concerning the issues and differences in videos compatibility, MSP and network operators believe that the video quality is a more important task in the multimedia processing systems and introduce the video QA methods. QA measures video signals, compression level, and video quality degradation. Moreover, video QA methods can be measured both objectively using mathematical models and subjectively involving user perceptions.

In this paper, our focus is on the object video quality (OVQ) model reflecting the fact that quality models can be measured objectively but do not work together because OVQ is free from human interpretations, and it is considering a part in subjective quality assessment techniques. The OVQ is a dependent variable of mathematical models in which several independent variables (e.g., video coding parameters) are well worked against the subjective assessment using statistical models and regression techniques. The OVQ models can be defined through the video information such as the parameters available about the original video signal and received video or processed video signals. The three technical models, i.e., full reference (FR), reduced reference (RR), and no-reference (NR) models, are mostly used for video quality assessments, PSNR and video quality measurement (VQM) [7].

Generally, the reference model can measure the quality differences between the original video and the received video/processed video. The NR model makes efforts to work for distorted videos in the absence of original reference video, but the NR model approach is a less accurate method as compared to the FR model approach. The FR model considers the degradation of video quality and evaluates it more accurately as compared to human-perceived assessments. The reference method is more complicated and most reliable for video quality assessment that requires the availability of the original video sequence to compute a video quality before transmitting the video coding. Therefore, in most of the situations, the OVQ and FR model requires more computational efforts [7-9].

However, Internet service providers (ISPs) face several multimedia services issues while transmitting $\mathrm{HD}$ videos. Guaranteed QoS and improved quality (video) of user experience is a challenge for service providers because $\mathrm{HD}$ videos contain high data size and occupy more storage at cloud site, and during uploading, it requires high network bandwidth to transfer these files on the cloud server. This problem leads to a critical issue of service delivery, and it is essential to the analysis of video quality. SC is the stand-alone application where a variety of multimedia services is offered to the users such as video sharing and video posting. These services are generally used by end-users to share their recorded videos on social media [2]. End users share and upload their HD video on different SC, but the original video compressed by SC does not form the original video because the social cloud stores the video by using predefined compression techniques of different video codecs [3-5].

Our main contributions are to present the real video streaming analysis and compare results of video quality with reference model metrics. The major objectives include the following: (i) Evaluation of three different video resolutions (144p, 240p, and 360p) and their comparison with $\mathrm{SC}$ and original videos

(ii) Impact of PSNR and VQM in SC videos

(iii) Evaluation of mean square error (MSE), MSAD, and Delta and data distortions in SC

(iv) Impact of SC videos and different levels of video degradations

The rest of this paper is organized as follows. Section 2 is related to work on existing objective video quality. Section 3 proposes techniques and preliminary experiments. Section 4 provides detailed discussion and results. The paper is finally concluded in Section 5.

\section{Literature Review}

Researchers have introduced their work in the cloud services [10] and video quality metric [11] and have proposed various video quality models $[7,8,12]$ in different environments. The authors in [10] introduced cloud computing technology and proposed an efficient ESVP, Cloud Architecture, to demonstrate the performance of the mobile video stream. A cloud-assisted adaptive and efficient social video sharing method is also discussed. The proposed solution can store and share videos in the cloud because the authors only focused on improving the response time of live videos [13]. The cloud-based video live streaming cloud (VLSC) services proposed a scheduling method based on live video streaming to facilitate the video transcoding process and provide costefficient manners and satisfied the viewer QoS demands. In order to correlate with objective video quality, Appina and Channappayya [14] presented bit-stream based features to measure the full reference video quality metric (PSNR, PVQM, VQM, and VIF).

The video 560 bit-stream features are tested by highefficiency video coding (HEVC) and used the monotonic prediction of the number of objective quality metrics. The authors in [15] presented the work on full reference $3 \mathrm{D}$ video quality assessment and proposed FR stereoscopic VQA metric based on depth and motion subband coefficients of video frames. The coherence of the covariance matrix was performed, and the coherence scores were computed by applying off-the-shelf 2D FR image quality assessment metric on video frames.

Soundararajan and Bovik [16] discussed the objective quality assessment techniques for video conferencing coding and proposed the Gaussian mixture model-based peak signal-to-noise ratio (GMM-PSNR). The work evaluated the quality of video conferencing coding. Multimedia file format and codec are widely used in video communication systems based on the related work of wireless network and proposed algorithms for a multimedia file format (MPEG4, FLV, and 3GP). The authors used the ratio of signal power to the noise power (SNR) and peak signal-to-noise ratio (PSNR) metrics to calculate the performance and differences of each file format. Results show that the different file formats vary in different qualities [16]. 
TABLE 1: Comparison of related work.

\begin{tabular}{|c|c|c|c|}
\hline Author & Techniques & Analysis & Description \\
\hline Wang et al. [20] & SSIM & VQEG phase I & $\begin{array}{c}\text { Performance as } \mathrm{PCC}=0969, \mathrm{SROCC}=0.966, \\
\mathrm{RMSE}=4.91 \text { and } \mathrm{OR}=1.16 .\end{array}$ \\
\hline Pessoa et al. [21] & DSIS & $\begin{array}{l}\text { MPEG-2 coded, five natural scene clipsITU- } \\
60 \text { PAL format }(57 \times 720) \text { interlaced, } 4: 2: 2 \\
\text { display ratio sampling to } 65 \text { sequences }\end{array}$ & Accuracy measured MAE less than $4 \%$. \\
\hline Watson et al. [22] & $\begin{array}{l}\text { DVQ, DCT, } \\
\text { DSCQS }\end{array}$ & Carphone sequence & Results show the RMSE $=14.61$. \\
\hline Xiao [23] & DCT, JNDs & Block and blur errors & $\begin{array}{c}\text { Methods show better performance than } \\
\text { RMSE }\end{array}$ \\
\hline \multirow{2}{*}{$\begin{array}{l}\text { Ndjiki-Nya et al. [24] } \\
\text { Chandler and Hemami } \\
{[25]}\end{array}$} & DMOS & - & Compare PCC, but better to PSNR. \\
\hline & VSNR & Live image database & $\begin{array}{l}\text { Accuracy measured of PCC and } \\
\text { SROCC }=0.889 \text { and } \mathrm{RMSE}=7.39\end{array}$ \\
\hline Laghri et al. [27] & MOS & Subjective assessment & $\begin{array}{l}\text { Measured the accuracy of different video file } \\
\text { formats }\end{array}$ \\
\hline
\end{tabular}

A comparison of objective video quality metrics was identified in mean opinion score (MOS) or DMOS by analyzing the correlation between subjective QoE, which were obtained from user test and perceived score by objective metrics. Results showed the insufficiency of subjective data and recommended to use different subjective procedures that revealed more comprehensiveness [17]. The quality assessment algorithm and a reduced reference VQ assessment is presented. A hybrid approach was used in statistical models to utilize the spatial and temporal differences by using a Gaussian scale mixture model. The proposed algorithm showed better performance PSNR for predicting the video quality. Other sides developed QoE predictive model for Internet videos that measured the user engagement parameters and relationships and showed delivery infrastructure that achieves $20 \%$ improvement in user engagement as compared to the other approaches [18].

However, online video streaming traffic and accuracy are estimated at $57 \%$ to $69 \%$, while playing video and user perceived video streaming services and providing user experience. The work measured QoE for online video streaming services. QoE metrics are used to optimize quality challenges but not in case of uploaded/downloaded videos from clouds [19].Video quality is estimated based on artificial neural network (ANN), and it adopted a neuro-fuzzy interface that predicted the MOS score. However, video quality is obtained from PSNR to MOS but did not perform subjective assessments [20]. The comparison of related work table is illustrated in Table 1 .

The quality of multimedia content under different video file formats and video resolutions is compared and analyzed. Subjective evaluation method was used to analyze the impact of video format on quality. The results show that MP4 video obtained the highest influences, but the author does not provide an objective video quality evaluation [26]. The subjective assessment method was used to analyze the impact of video formats on QoE. Results showed MOS that MP4 video obtained the highest rating for $720 p$ and $2160 p$, FLV for 240p, and WebM for 360p, but the authors did not provide objective video quality assessments [26].

Literature review reveals that few studies [26, 28] are conducted on subjective QoE video quality on social clouds, but the measurement of objective video quality with reference metrics (PSNR, VQM, MSE, MSAD, and Delta) in social clouds is yet not researched. To the best of authors' knowledge, this is the first study that deals with the problem of video quality and presents the outcomes of video quality using online video streaming and social media videos.

\section{Proposed Technique}

To examine the performance of video quality in SC, we preferred to use the three most popular social media clouds Facebook, VK, and WeChat in our experiments. Original videos were selected and taken from the YouTube site [29-31] and downloaded using source site [32]. The original video contains three different video resolutions (144p, 240p, and 360p) and has the same (.mp4) file format uploaded on social clouds.

The technical parameters of the original video were recorded before uploading the original video on SCs because the social clouds automatically compress the original video parameters according to their predefined compression algorithms and reduce the storage size. In addition, the original and resulting videos were compared and examined to know the full reference metric by using VQMT simulation tool. The proposed design is given in Figure 1 and the relevant technical parameters are given in Table 2.

The peak signal-to-noise ratio (PSNR) metric is widely used in the field of signal processing because it is a maximum ratio of power between signals to noise. PSNR average (Avg) value is commonly used in the practice of video processing, where video quality and loss compression codecs are considered for testing of video quality or improving the video codec. The higher and lower value of PSNR indicates the reconstructed video quality. If the value of PSNR in video compression is between $30 \mathrm{~dB}$ to $50 \mathrm{~dB}$ and provides 8 bits of depth, then $50 \mathrm{~dB}$ PSNR is better. This metric is also useful to measure video codec standards (MPEG-4, H.263, and H.264). Therefore, this metric is only valid when it applies to compare the results between the same codec, the same codec type, and the same corresponding video contents. Generally, PSNR is equivalent to MSE, but it is easily defined as compared to MSE because of the scale of the technique. It can be calculated by the equation given in [33]. 


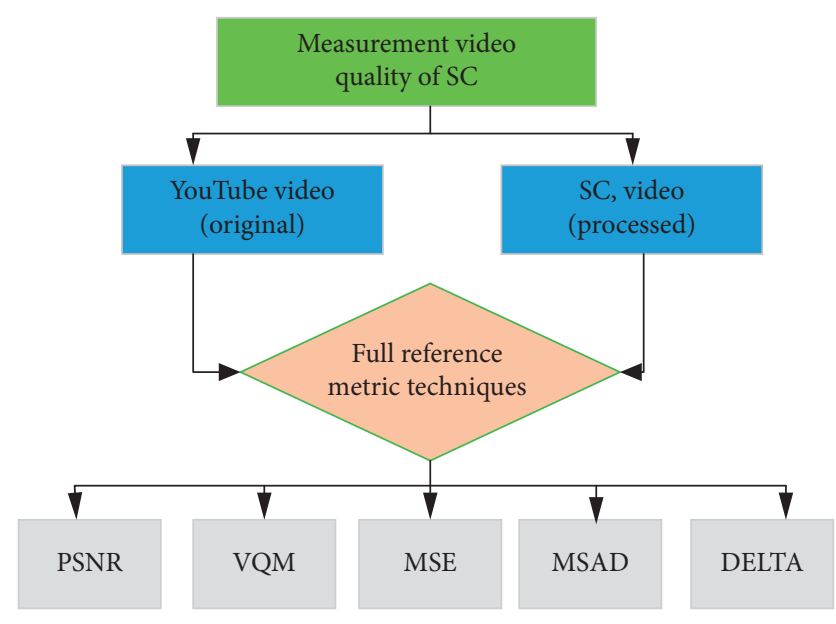

FIGURE 1: Flowchart of the proposed techniques.

Table 2: Parameters for proposed techniques.

\begin{tabular}{lc}
\hline Parameters & Description \\
\hline MaxErr & Maximum value of color components \\
$w$ & Video width \\
$h$ & Video height \\
MSE & Mean squared error \\
DMAD & Delta and mean absolute difference \\
$\left(x_{i}\right)$ and $\left(y_{i}\right)$ & Two difference signal \\
$Y_{i j}<x_{i j}$ & Red color \\
$x_{i j}>Y_{i j}$ & Green color \\
$1 / N$ & Length of the signal \\
\hline
\end{tabular}

$$
\text { PSNR }=10 . \log _{10} \frac{\operatorname{Max}_{\operatorname{Err}^{2} \times w \times h}}{\sum_{i=1, j=1}^{w, h}\left(x_{i, j}-y_{i, j}\right)^{2}}
$$

Calculate the average PSNR for sequence video and evaluate MSE average for all frames using the ordinary equation of PSNR that can be presented as

$$
\text { PSNR }=10 . \log _{10} \frac{\text { Max Err }^{2}}{\text { MSE }} .
$$

VQM is generally used for objective video quality measurements. This metric evaluates the video streams between original video to processed video and includes features with spatial-temporal alignments, valid region-estimations, and gain-level offset calculations. VQM calculation can extract perception-based features to compute the video quality parameters and generate more accurate predictions of human-perceived video quality [34].

$$
\mathrm{VQM}=\frac{1}{1+e^{0.17 *(\mathrm{PSNR}-25.66)}}, \quad 10 \leq \mathrm{PSNR} \leq 55
$$

The computed MSE metric is the simplest metric to compute differences between two videos and compare video signals. It can be calculated as

$$
\operatorname{MSE}=\frac{1}{N} \sum_{i=1}^{N}\left(\left(x_{i}\right)-\left(y_{i}\right)\right)^{2} .
$$

Delta and mean absolute difference (MSAD) metrics are also the simplest way to calculate the mean differences of the color $(R, G$, and $B)$ components in the corresponding values of the video, and this metric is mostly used for filters and testing codecs. Therefore, the VQM supports all features of MSE, Delta, and MSAD. MSE and Delta can be calculated by

$$
d(x, y)=\frac{\sum_{i=1, j=1}^{m, n}\left(x_{i, j}-y_{i, j}\right)^{2}}{m \times n},
$$

where $Y_{i j}<x_{i j}$ corresponds to red color and $x_{i j}>Y_{i j}$ corresponds to the green color in Delta metrics. MSAD metric is calculated by

$$
d(x, y)=\frac{\sum_{i=1, j=1}^{m, n}\left|x_{i, j}-y_{i, j}\right|^{2}}{m \times n} .
$$

\section{Results and Discussion}

This section presents the detailed results and analysis of video quality; furthermore, it analyzes three SCs (Facebook, $\mathrm{VK}$, and WeChat) and low-quality videos (144p, 240p, and $360 \mathrm{p})$. Selected videos are uploaded to SC to assess differences in video services and quality.

4.1. Original Video Parameters. To collect the objective video quality analysis and results, in the first step, we measured the original video parameters by using VLC (Video LAN Client) media player (version 2.2.1) [34]. The VLC extracted the detailed information of each video file in technical parameters (e.g., frame width/height, data rate, total bitrate, frame rate, audio bitrate, storage size, playing duration, and video file format). The screenshots of original videos and technical parameters are given in Figure 2 and Table 3.

4.2. Social Cloud Video Quality with Reference Metrics. In this section, the interpretation with reference metrics and results are explained. Hence, the five reference metrics (PSNR, MSE, MSAD, Delta, and VQM) are considered. The Moscow State University (MSU) video quality measurement tool (VQMT) software (version 11.0) is preferred for analysis. This software is specially used for testing video codecs and to perform a comparative analysis of objective video quality using reference and no-reference metrics. For interpretation of each parameter, it is necessary to know the values of the metrics, i.e., either social videos provide better quality or not to the end-users. Each frame can measure PSNR in video sequence, which corresponds to the higher/lower values of an average (Avg). The higher value $(\operatorname{Avg}=100)$ is better for the same frames in PSNR. Furthermore, lower values $($ Avg $=0)$ for VQM, MSE, and MSAD are better for equal frames, and lower Delta value $($ Avg $=0)$ indicates equal frames, i.e., both are signed $(+,-)$ values in mean deviation, which means lower absolute values are better. The parameters of the reference metric are listed in Table 4.

We uploaded 144p video on the Facebook social cloud, and analysis of Facebook Video 144p is presented in Figure 3. Results reveal that Facebook provides low quality 


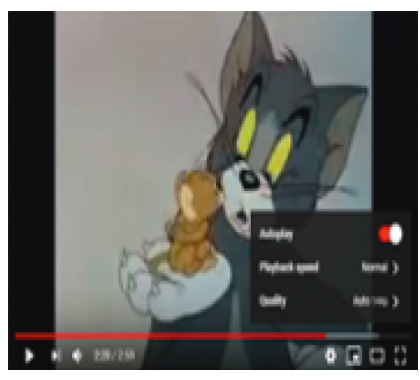

(a)

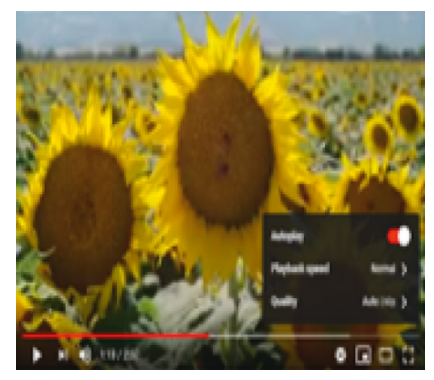

(b)

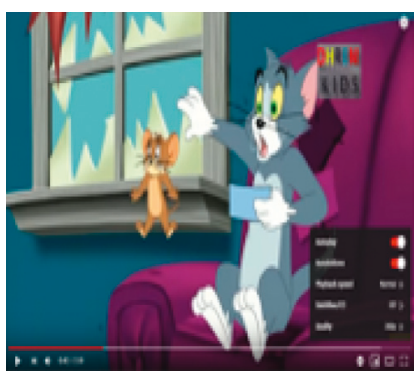

(c)

Figure 2: 144p, 240p, and 360p original video screenshots. (a) Original video 144p. (b) Original video 240p. (c) Original video 360p.

TABLE 3: Technical parameters of original video.

\begin{tabular}{lccc}
\hline Technical parameters & Video $144 \mathrm{p}$ & Video 240p & Video 360p \\
\hline Frame width & 256 & 426 & 640 \\
Frame height & 144 & 240 & 360 \\
Data rate & $177 \mathrm{kbps}$ & $289 \mathrm{kbps}$ & $433 \mathrm{kbps}$ \\
Total bitrate & $274 \mathrm{kbps}$ & $385 \mathrm{kbps}$ & $530 \mathrm{kbps}$ \\
Frame rate & $29 \mathrm{frames} / \mathrm{sec}$ & 29 frames/sec & $29 \mathrm{frames} / \mathrm{sec}$ \\
Audio bitrate & $96 \mathrm{kbps}$ & $96 \mathrm{kbps}$ & $96 \mathrm{kbps}$ \\
Storage size & $5.90 \mathrm{MB}$ & $7.27 \mathrm{MB}$ & $11.3 \mathrm{MB}$ \\
Playing length & $00: 02: 59$ & $00: 02: 37$ & $00: 02: 59$ \\
Video file format &. $\mathrm{mp} 4$ &. $\mathrm{mp} 4$ &. $\mathrm{mp} 4$ \\
\hline
\end{tabular}

TABle 4: Parameters of reference metric.

\begin{tabular}{lcr}
\hline Parameters & Quality & Value \\
\hline PSNR & Best & 100 (higher value) \\
VQM & Better & 0.00 (lower value) \\
MSE & Better & 0.00 (lower value) \\
MSAD & Better & 0.00 (lower value) \\
Delta & Better & 0.00 (lower value) \\
\hline
\end{tabular}

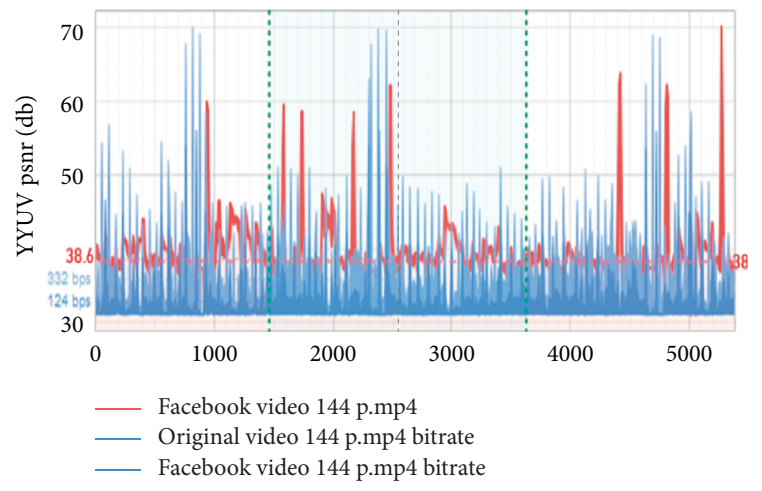

(a)

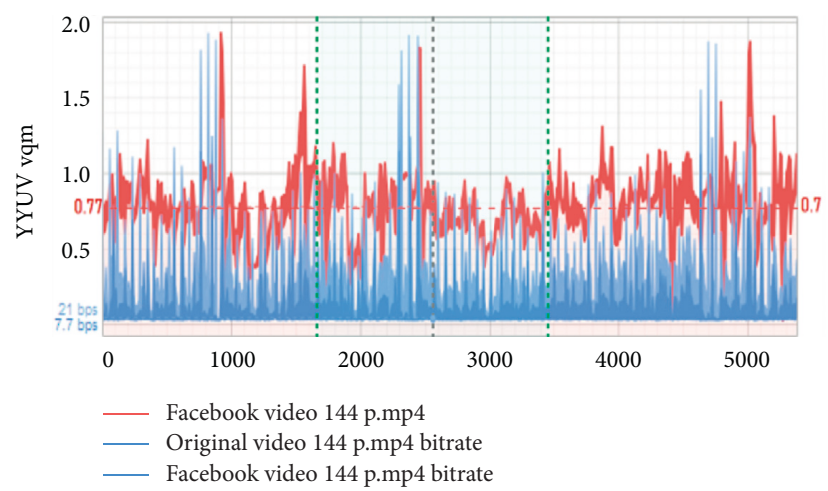

(b)

FIGURE 3: Continued. 


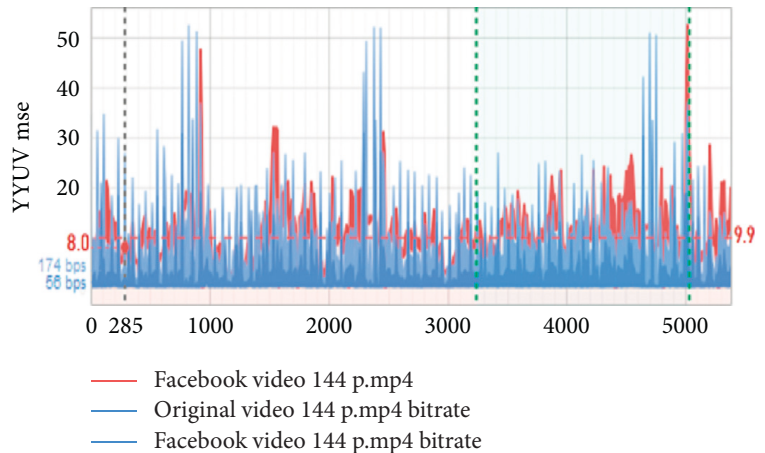

(c)

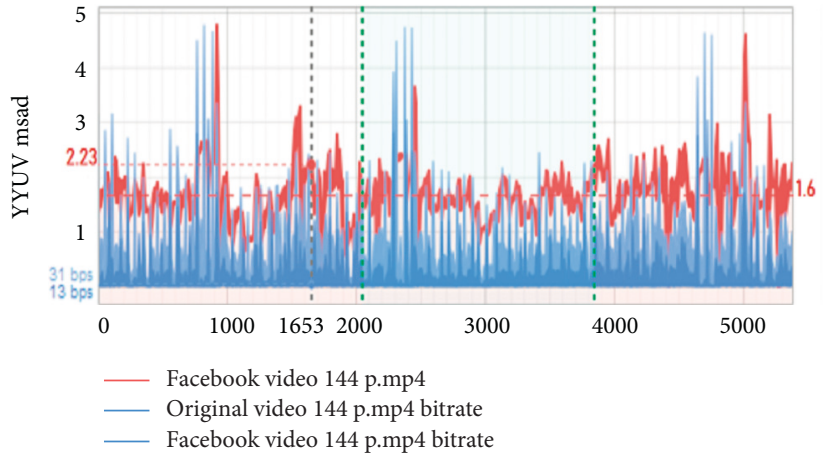

(d)

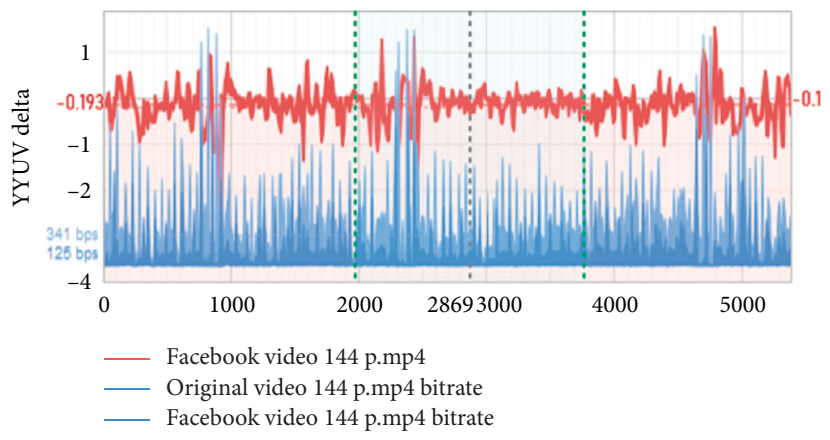

(e)

Figure 3: (a, b, c, d, e) Comparison of original video and Facebook 144p quality metrics results. (a) PSNR average value $=38.155602$. (b) $\mathrm{VQM}$ average value $=0.770144$. (c) MSE average value $=9.943065$. (d) MSAD average value $=1.666160$. (e) Delta average value $=-0.1384996772$.

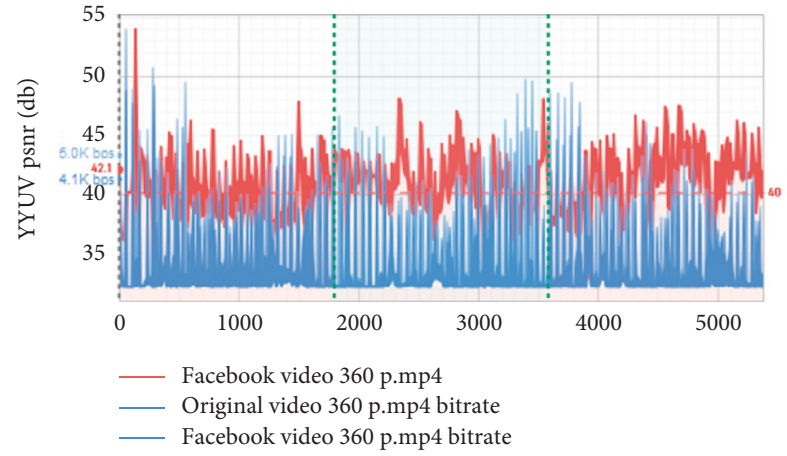

(a)

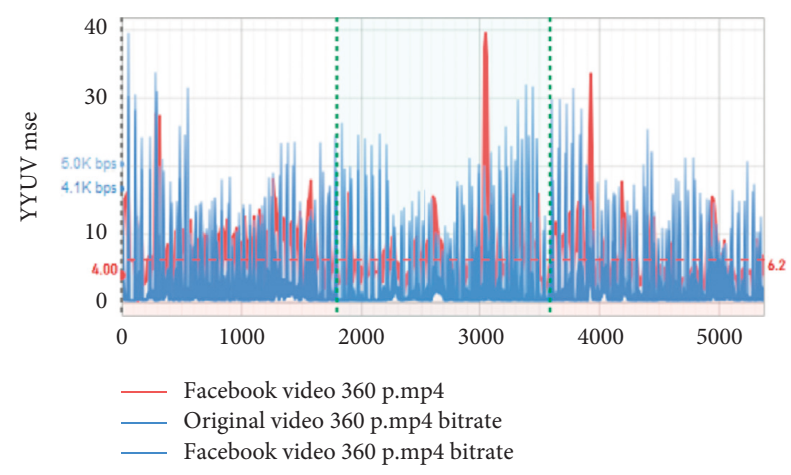

(c)

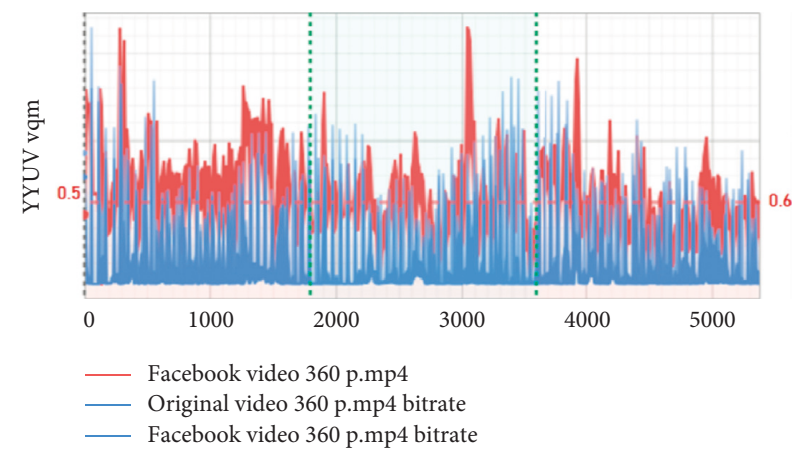

(b)

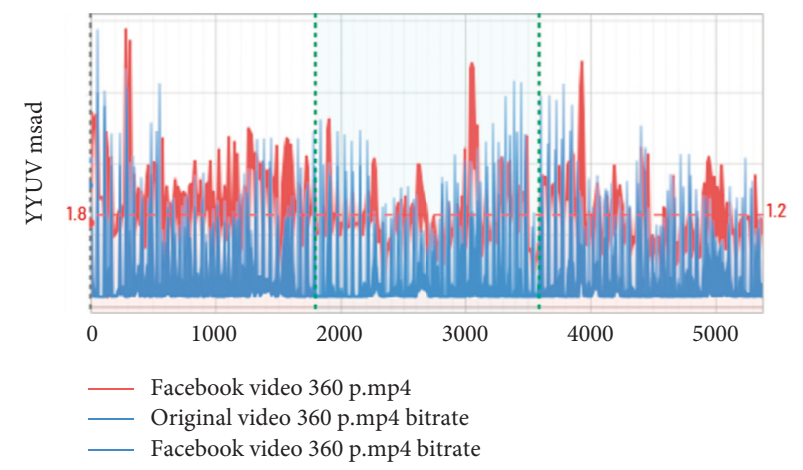

(d)

Figure 4: Continued. 


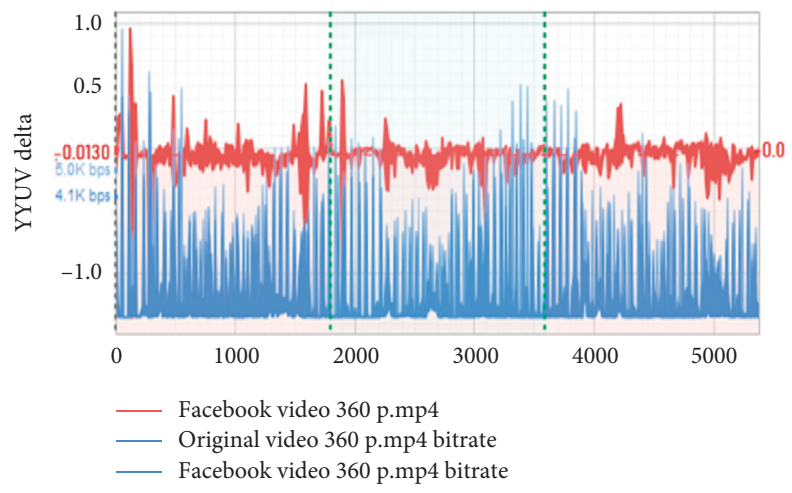

(e)

FIGURe 4: (a, b, c, d, e) Comparison of original video and Facebook 360p quality metrics results. (a) PSNR average value $=40.156540 .(b)$ VQM average value $=0.648435$. (c) MSE average value $=6.272291$. (d) MSAD average value $=1.291984$. (e) Delta average value $=-0.051095$.

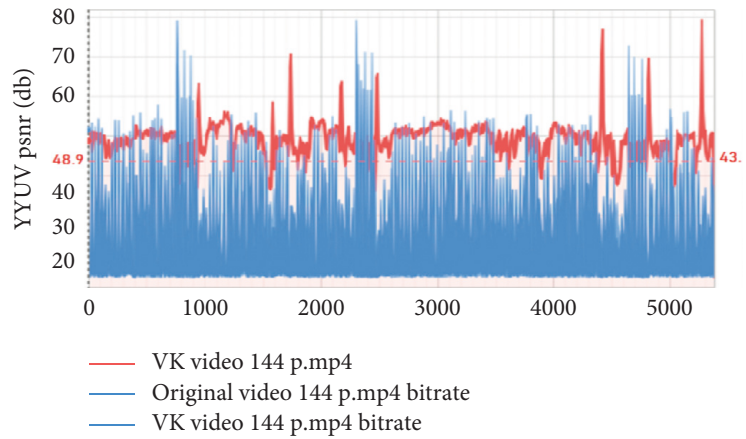

(a)

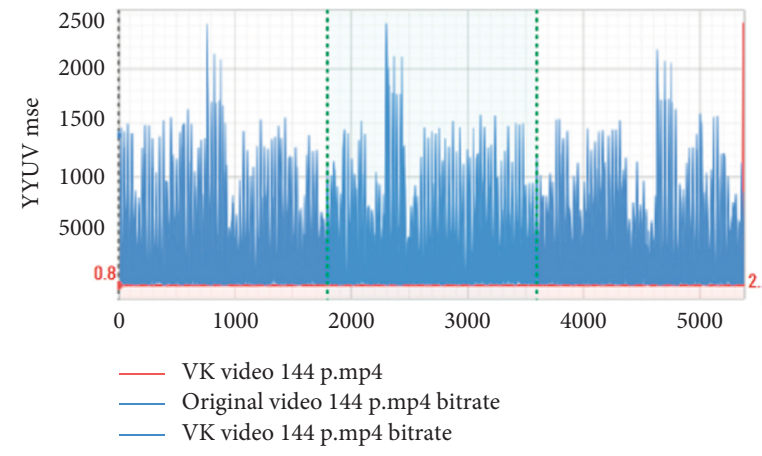

(c)

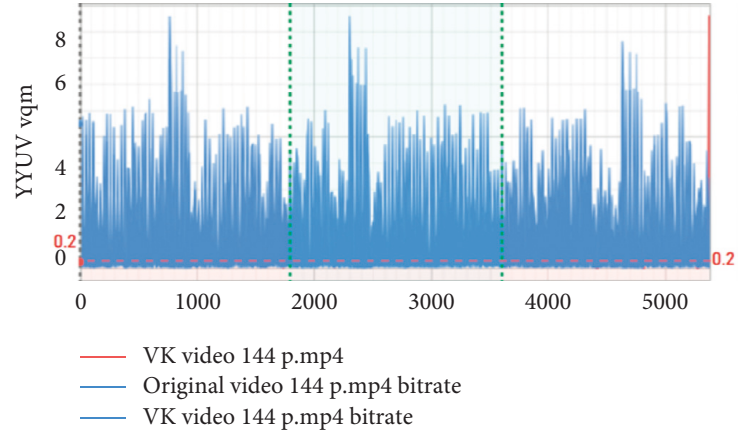

(b)

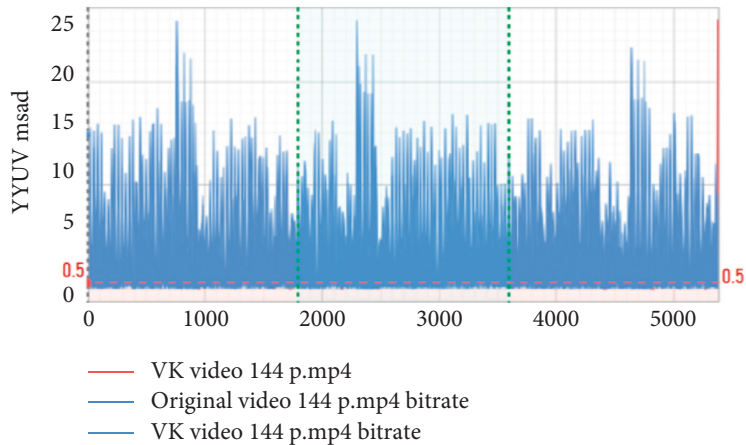

(d)

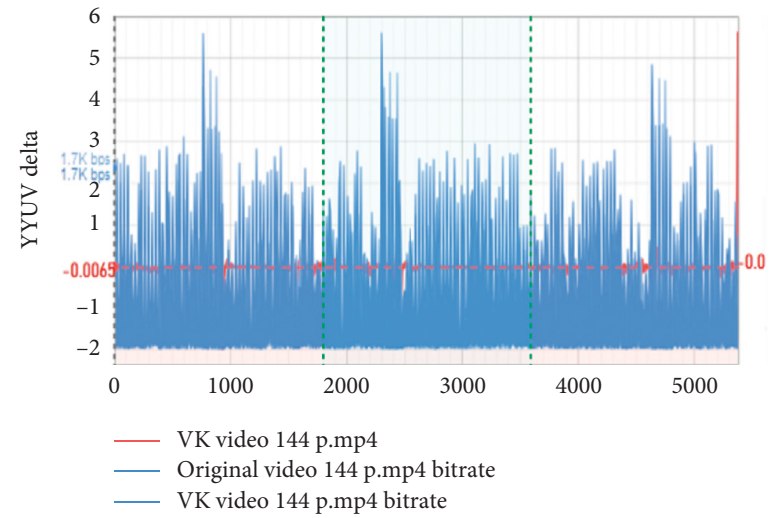

(e)

Figure 5: (a, b, c, d, e) Comparison of original video and VK 144p quality metrics results. (a) PSNR average value $=48.9$. (b) VQM average value $=0.250$. (c) MSE average value $=0.847$. (d) MSAD average value $=0.520$. (e) Delta average value $=-0.00659$. 


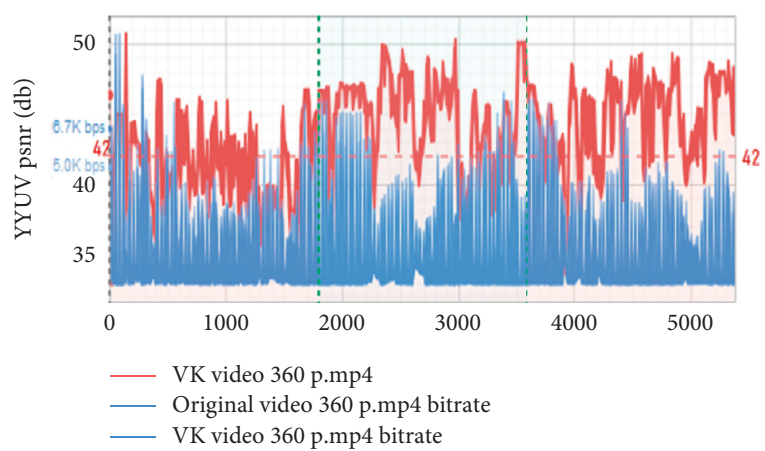

(a)

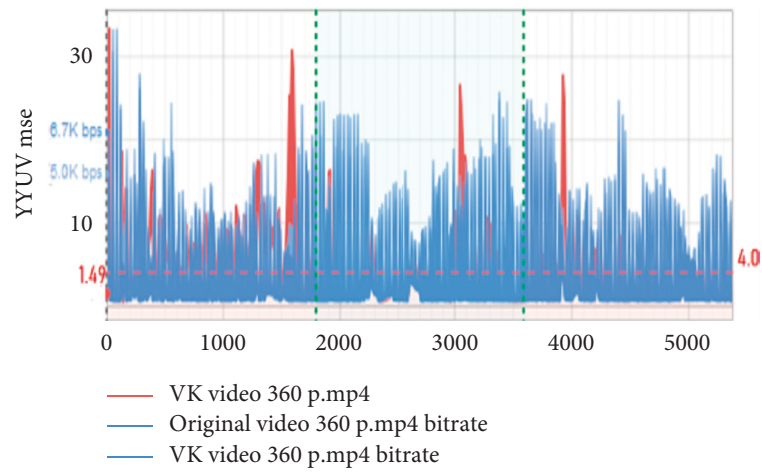

(c)

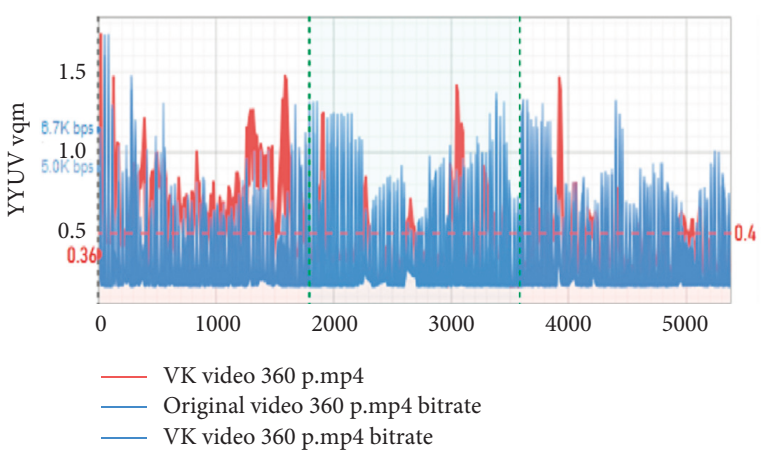

(b)

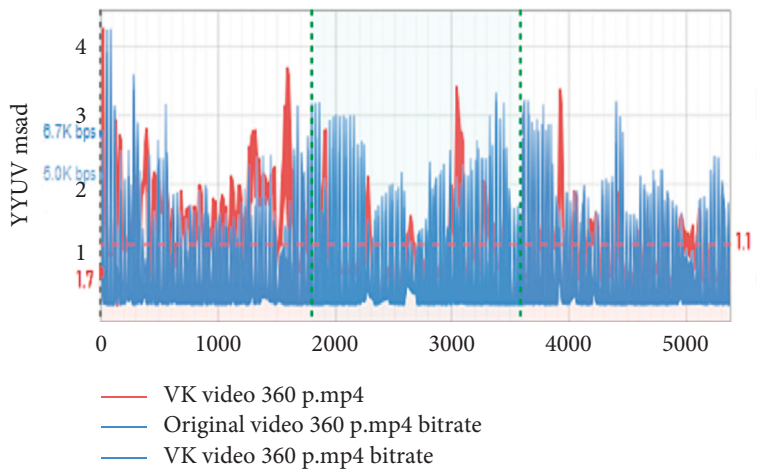

(d)

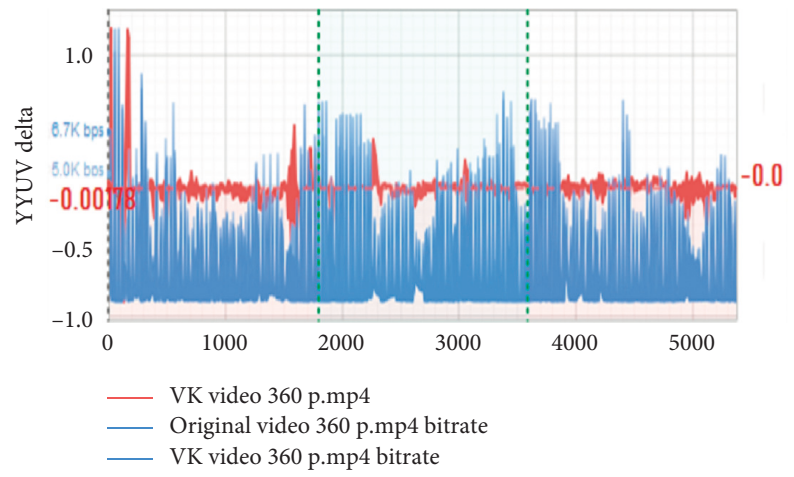

(e)

FIgure 6: (a, b, c, d, e) Comparison of original video and VK 360p quality metrics results. (a) PSNR average value $=46.4$. (b) VQM average value $=0.368$. (c) MSE average value $=1.49$. (d) MSAD average value $=0.718$. (e) Delta average value $=-0.00178$.

and shows that Avg (38.155602) PSNR is less than 100 PSNR quality, VQM metrics (Avg $=0.770144<0)$, MSE metric value $(\mathrm{Avg}=9.943065)$, MSAD (Avg=1.666160), and Delta metrics (Avg $=-0.138500)$. Facebook Video 240p is not processed by VQMT tool because video resolution scale is decreased to $(400 \times 224)$ and is comparatively different from the default original video.

Facebook video 360p results are shown in Figure 4; PSNR value Avg $=40.156540$, with original $5.0 \mathrm{kbps}$ bitrate as compared to $4.1 \mathrm{kbps}$ Facebook bitrate is observed. Furthermore, VQM metric was noticed, Avg $=0.648435$, lower than zero and MSE value, i.e., Avg $=6.272291$. The MSAD value, i.e., $\operatorname{Avg}=1.291984$, and Delta value were observed to be negative, i.e., $\operatorname{Avg}=-0.051095$.

Figure 5 shows the results of VK video 144p. We observed low resolution video quality that VK decreases the
PSNR $($ Avg $=48.9<100)$, VQM metric $($ Avg $=0.250)$, MSE $($ Avg $=0.847), \quad$ MSAD $\quad($ Avg $=0.520), \quad$ and Delta $($ Avg $=-0.00659)$ with $1.7 \mathrm{kbps}$ bitrate. Furthermore, VK video $240 \mathrm{p}$ is also not processed by VQMT tool because the resolution of video is decreased $(320 \times 180)$ and different from the default values.

The next observation of VK Video 360p is shown in Figure 6. The results show that PSNR value is $\mathrm{Avg}=46.4<100$, which is $5.0 \mathrm{kbps}$ lower than the original 6.7kbps. Furthermore, it is shown that all metrics are greater than zero such as VQM $($ Avg = 0.368), MSE (Avg= 1.49), MSAD (Avg=0.718), and Delta (Avg $=-0.00178)$.

We repeat the same for the next social cloud WeChat for video $144 \mathrm{p}$. WeChat provided the best quality metrics; we observed that PSNR value was $\mathrm{Avg}=100$, with bitrate $1.7 \mathrm{kbps}$ as compared to original bitrate $1.7 \mathrm{kbps}$, and the rest 


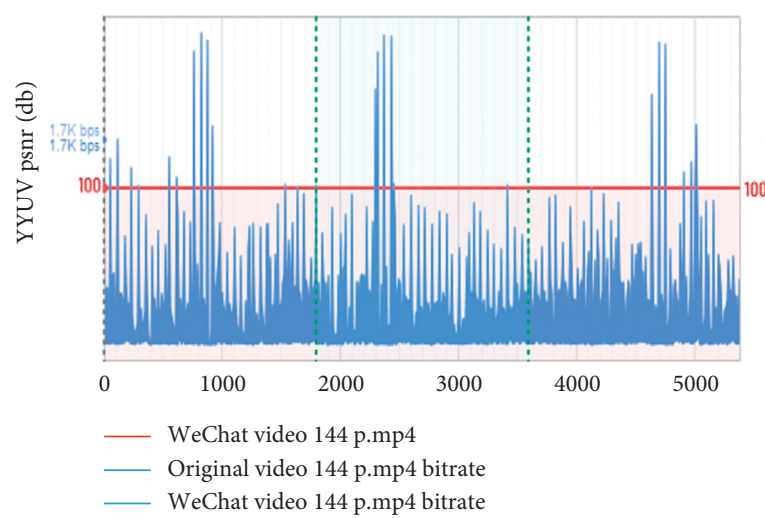

(a)

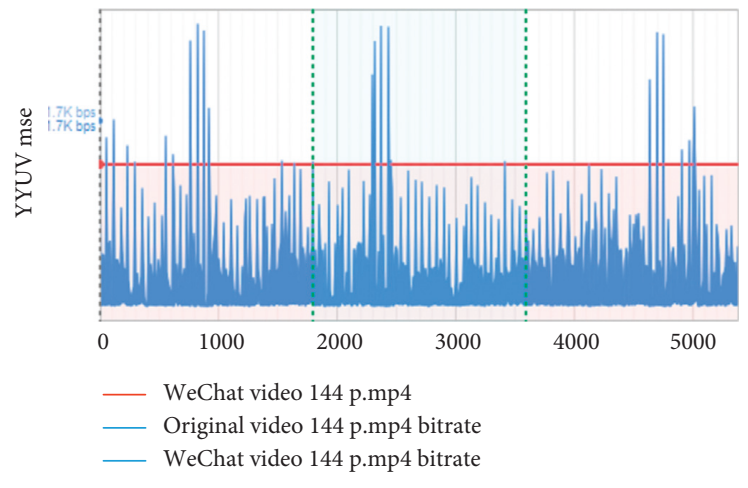

(c)

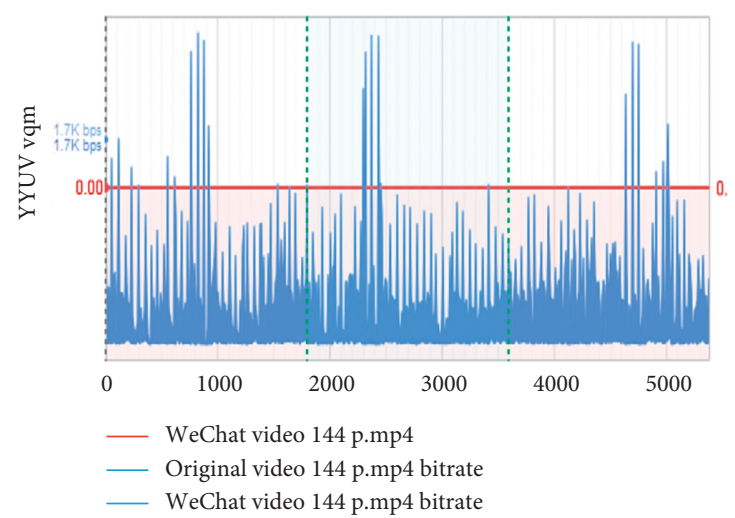

(b)

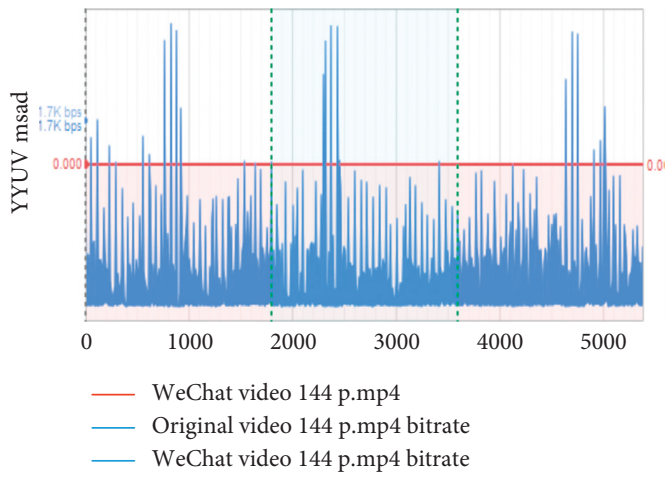

(d)

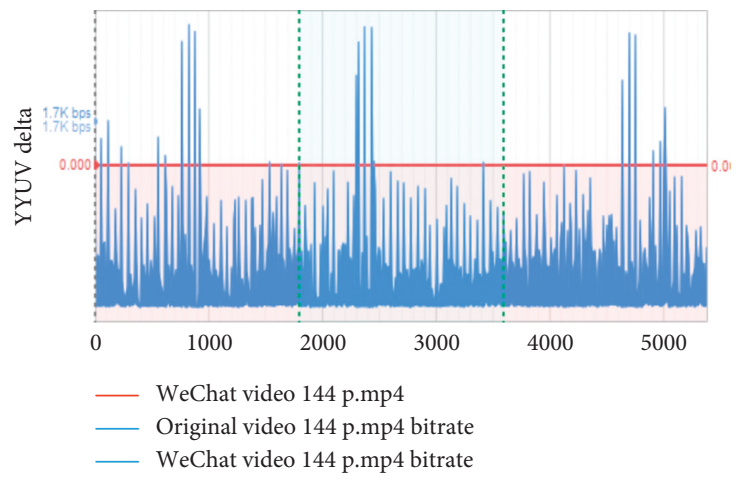

(e)

Figure 7: (a, b, c, d, e) Comparison of original video and WeChat 144p quality metrics results. (a) PSNR average value $=100 .(\mathrm{b})$ VQM average value $=0.000$. (c) MSE average value $=0.000$. (d) MSAD average value $=0.000$. (e) Delta average value $=0.000$.

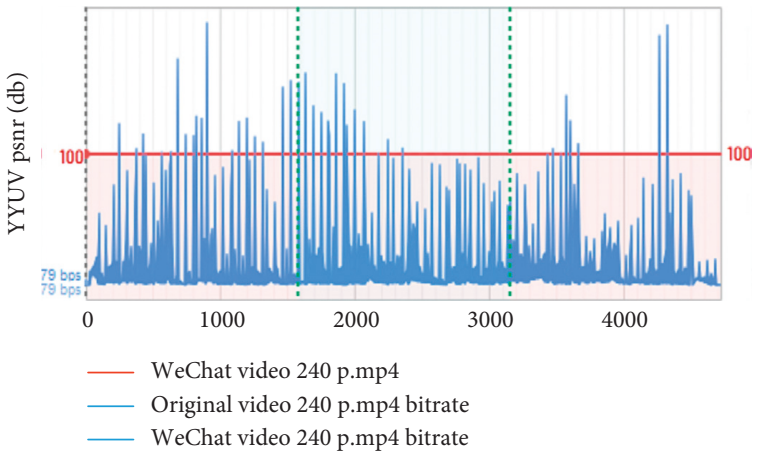

(a)

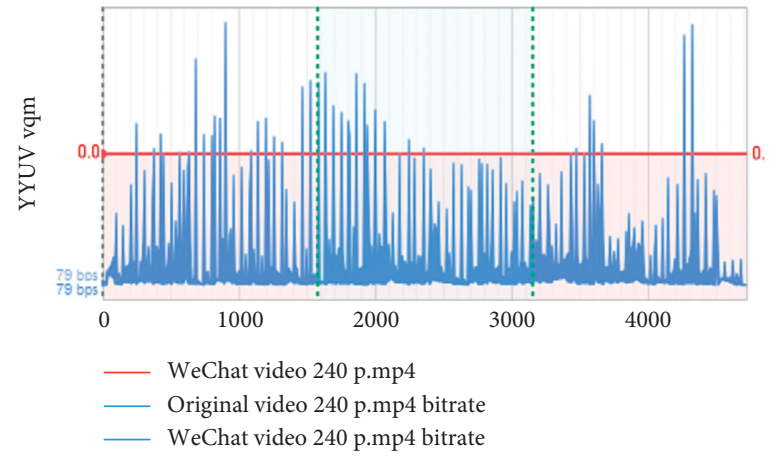

(b)

FIgUre 8: Continued. 


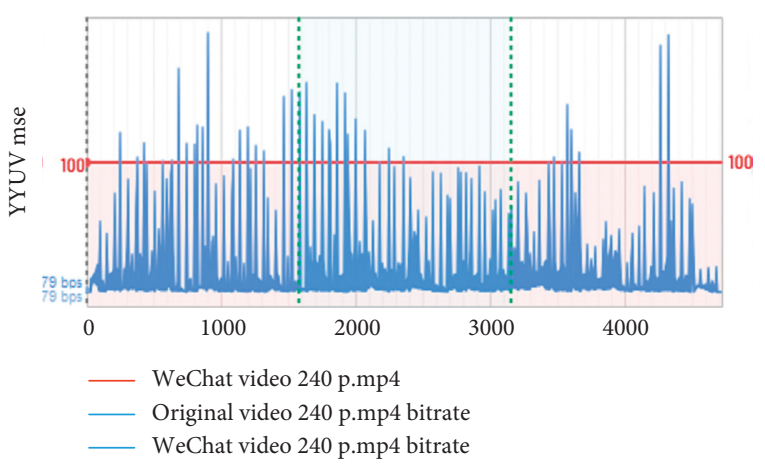

(c)

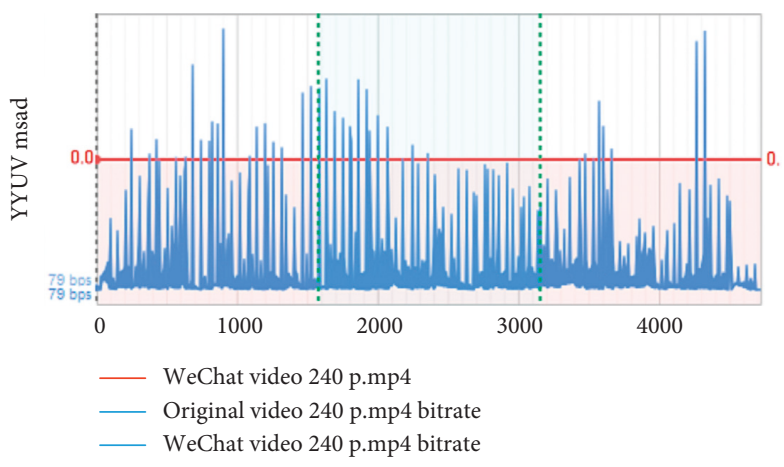

(d)

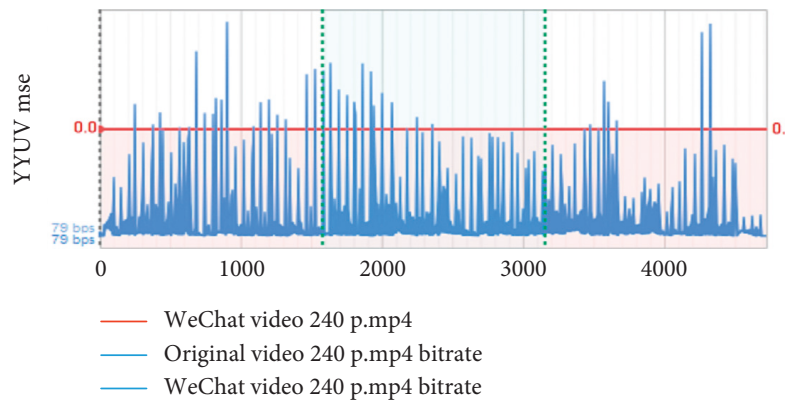

(e)

FIgure 8: (a, b, c, d, e) Comparison of original video and WeChat 240p quality metrics results. (a) PSNR average value = 100. (b) VQM average value $=0.000$. (c) MSE average value $=0.000$. (d) MSAD average value $=0.000$. (e) Delta average value $=0.000$.

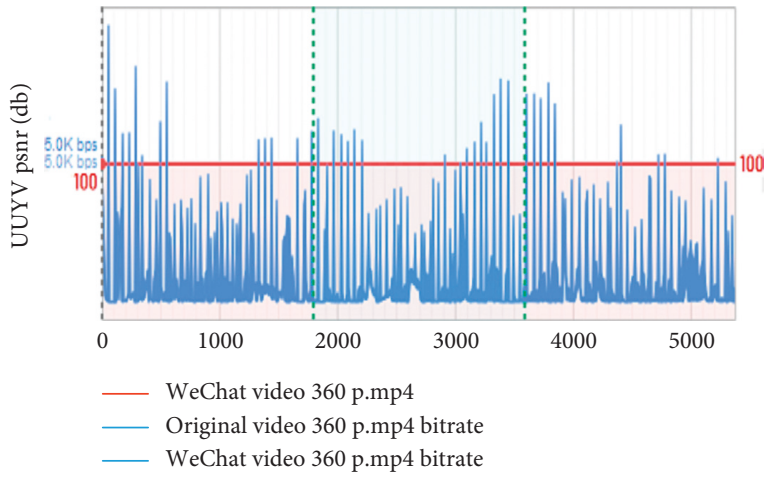

(a)

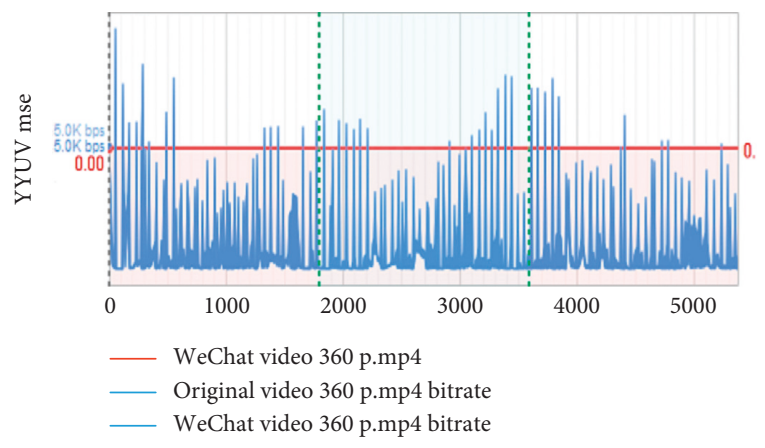

(c)

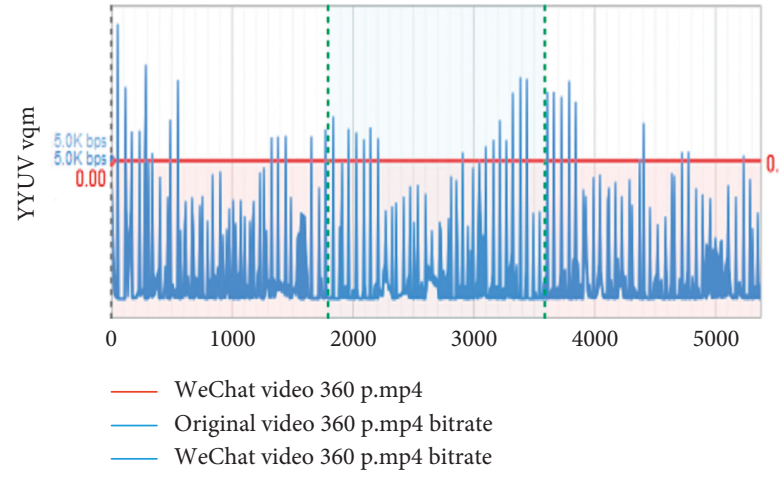

(b)

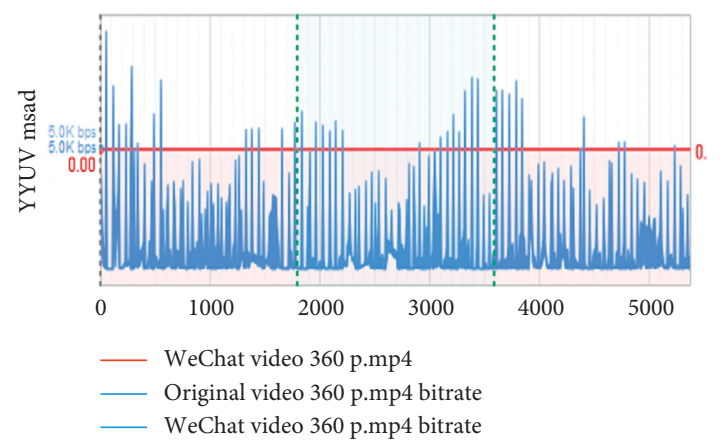

(d)

Figure 9: Continued. 


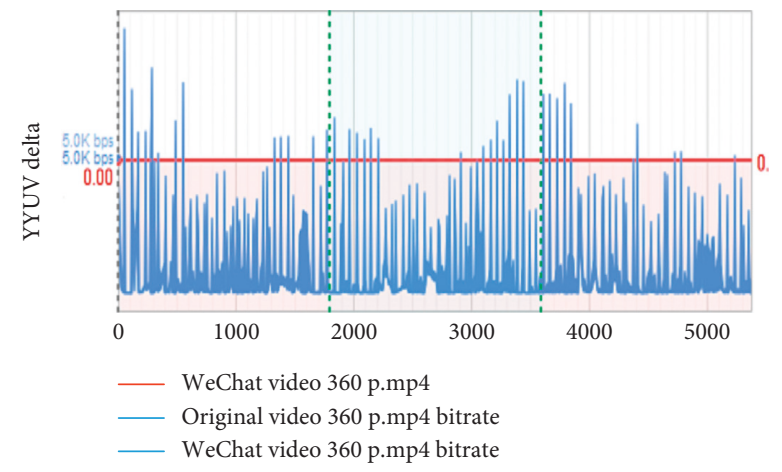

(e)

Figure 9: (a, b, c, d, e) Comparison of original video and WeChat 360p quality metrics results. (a) PSNR average value $=100$. (b) VQM average value $=0.000$. (c) MSE average value $=0.000$. (d) MSAD average value $=0.000$. (e) Delta average value $=0.000$.

TABLE 5: Consolidated comparison of average values of social clouds.

\begin{tabular}{lccccccccc}
\hline \multirow{2}{*}{ Metrics } & \multicolumn{3}{c}{ Video 144p } & \multicolumn{3}{c}{ Video 240p } & \multicolumn{3}{c}{ Video 360p } \\
& Facebook & VK & WeChat & Facebook & VK & WeChat & Facebook & VK & WeChat \\
\hline PSNR & 38.155602 & 43.635052 & 100 & N/A & N/A & 100 & 40.15654 & 42.041149 \\
VQM & 0.770144 & 0.277658 & 0.000 & N/A & N/A & 0.000 & 0.648435 & 0.497257 & 0.000 \\
MSE & 9.943065 & 2.815627 & 0.000 & N/A & N/A & 0.000 & 6.272291 & 4.064112 & 0.000 \\
MSAD & 1.66616 & 0.594970 & 0.000 & N/A & N/A & 0.000 & 1.291984 & 1.123499 & 0.000 \\
Delta & -0.1384997 & -0.021268 & 0.000 & N/A & N/A & 0.000 & -0.051095 & -0.020238 & 0.000 \\
\hline
\end{tabular}

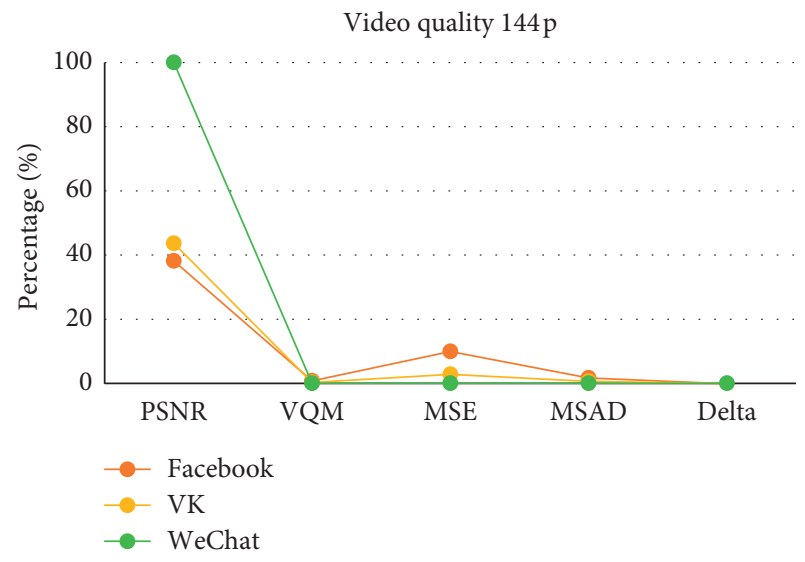

FIGURE 10: Comparison of $144 \mathrm{p}$ video quality values of three SCs.

of the metric value was $(\mathrm{Avg}=0.000)$. WeChat $(144 \mathrm{p})$ results are shown in Figure 7.

The second video of WeChat $240 \mathrm{p}$ analyzed the same results as compared to WeChat $144 \mathrm{p}$. The PSNR value was $($ Avg $=100)$ with bitrate $2.6 \mathrm{bps}$ as compared to original bitrate of $2.6 \mathrm{bps}$, and the rest of the metric value was $(\mathrm{Avg}=0.000)$. WeChat $240 \mathrm{p}$ results are shown in Figure 8 .

Further, we evaluate WeChat 360p video. The results show that PSNR value was $(\operatorname{Avg}=100)$, and VQM, MSE, MSAD, and Delta metric values were $($ Avg $=0.000)$ with bitrate $5.0 \mathrm{kbps}$ as compared to original bitrate of $5.0 \mathrm{kbps}$,

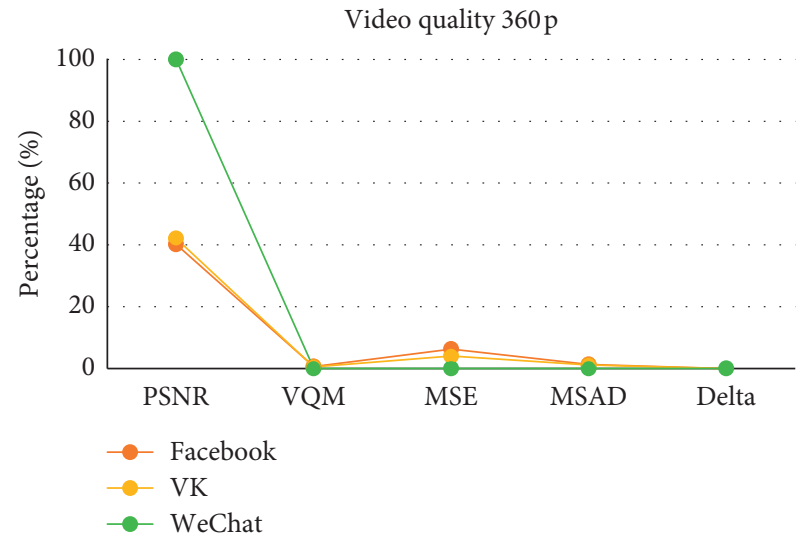

FIGURE 11: Comparison of 360p video quality values of three SCs.

and the rest of the metric value was $(\mathrm{Avg}=0.000)$. The extracted WeChat 360p metrics results are given in Figure 9.

The conclusion that we can make from the consolidated comparison results is presented in Table 5 . The results show that in video $144 \mathrm{P}$, Facebook video decreases as PSNR $($ Avg $=61.84 \%)$ and VK (Avg $=56.36 \%)$. In addition, Facebook video $240 \mathrm{P}$ with decreased video parameters cannot extract data and mismatch video attributes, and Facebook video $360 \mathrm{P}$ reduces PSNR (Avg $=59.84 \%$ ) and VK (Avg $=57.95 \%)$. As compared to all WeChat videos, PSNR value was $(\operatorname{Avg}=100)$. It is evident that WeChat 
provides the best quality of videos to the end-users. Furthermore, the comparison of video quality $144 \mathrm{p}$ and $360 \mathrm{p}$ of three social cloud results is shown in Figures 10 and 11.

\section{Conclusion}

We used Intel ${ }^{\circledR}$ core $^{\mathrm{TM}} \mathrm{i} 7-4790$ CPU @3.60GH, RAM 16 GB, and 64 bit OS type. In this paper, we explore an objective video quality assessment technique which is based on reference metrics (PSNR, VQM, MSE, MSAD, and Delta) and is analyzed and compared with the social cloud hosting and video services. While many researchers worked on subjective QoE, objective QoE video quality of SC is never considered by anyone in past research. Our focus is on analyzing video distortion and added noise in videos while uploading on social clouds to assess which video parameters have higher impact on objective QoE. This paper conducted experiments on free social cloud videos. The low-resolution video (144p, 240p, and 360p) was selected from YouTube and was uploaded on Facebook, VK, and WeChat for measuring the social cloud video quality and providing QoS/QoE user satisfaction level. These results provided an assessment of video quality, in which social clouds suffered due to a high compression level. Results show that decrease of full reference metrics has a higher impact on video quality, i.e., the results show that for video $144 \mathrm{p}$, the average PSNR of Facebook decreased by $61.84 \%$, while the average PSNR of VK decreased by $56.36 \%$. Video $360 \mathrm{p}$ showed that the average PSNR of Facebook was affected $(\operatorname{Avg}=59.84 \%)$, and the average PSNR of VK was affected (Avg $=57.95 \%)$. The other indicators (VQM, MSE, MSAD, and Delta) were also affected, compressing video at 144p, 240p, and 360p because the average PSNR value was inefficient. WeChat shows that the average peak signal-to-noise ratio of all videos is $($ Avg $=100)$ PSNR, which has no impact on other indicators and is the best video quality. We prove in results that Facebook and VK compressed more videos and provided low quality of video, but increased user QoE. Therefore, we can conclude that WeChat provides a high quality of services that ensure user satisfaction. This research will be helpful for service providers to pay concentration and improve multimedia services in the future.

\section{Data Availability}

The data used to support the findings of the this study are included within the article.

\section{Conflicts of Interest}

The authors declare no conflicts of interest.

\section{Authors' Contributions}

Sajida Karim and Hui He conducted research to write the paper and A. R. Junejo and Mariyam Sattar reviewed the paper to set context and contributed as experts of the field.

\section{Acknowledgments}

This study was supported by the National Key R\&D Program of China under grant no. 2017YFB803304 and the National Science Foundation of China (NSFC) under grant nos. 61872110 and 61672186.

\section{References}

[1] A. Periyannan, E. Weber, M. Grupenhoff et al., "Provision of video conferencing services using a micro pop to extend media processing into enterprise networks," Blue Jeans Network Inc., San Jose, CA, USA, U.S. Patent, 2019.

[2] S. B. Calo, D. M. Freimuth, V. Franck, M. T. Le, and D. C. Verma, "Synchronized release of resources used in deferential cloud services," International Business Machines Corporation, Armonk, NY, USA, U.S. Patent Application 15/ 439, 2018

[3] A. Rashed and A. Chaturvedi, "Cloud computing characteristics and services: a brief review," International Journal of Computer Sciences and Engineering, vol. 7, no. 2, pp. 421-426, 2019.

[4] A. R. Junejo, Y. Shen, A. A. Laghari, X. Zhang, and H. Luo, "Molecular diagnostic and using deep learning techniques for predict functional recovery of patients treated of cardiovascular disease," IEEE Access, vol. 7, pp. 120315-120325, 2019.

[5] S. Santiago, A. Ojo, E. Estevez, and P. R. Fillottrani, "Towards a cognitive linked public service cloud," in Proceedings of the Working Conference on Virtual Enterprises, pp. 430-441, Springer, Cardiff, UK, September 2018.

[6] K. Vasileios, M. AVGeris, M. Michaloliakos, K. Tsagkaris, and S. Papavassiliou, "Utility decisions for QoE-QoS driven applications in practical mobile broadband networks," in Proceedings of the 2018 Global Information Infrastructure and Networking Symposium (GIIS), pp. 1-5, Thessaloniki, Greece, October 2018.

[7] ITU, User Requirements for Objective Perceptual Video Quality Measurements in Digital Cable Television, ITU-T Recommendation J.1443, Geneva, Switzerland, 2000.

[8] B. Ciubotaru, G.-M. Muntean, and G. Ghinea, "Objective assessment of region of interest-aware adaptive multimedia streaming quality," IEEE Transactions on Broadcasting, vol. 55, no. 2, pp. 202-212, 2009.

[9] P. Cuenca, L. Orozco-Barbosa, A. Garrido, and F. Quiles, "Study of video quality metrics for MPEG-2 based video communications," in Proceedings of the 1999 IEEE Pacific Rim Conference on Communications, Computers and Signal Processing (PACRIM 1999). Conference Proceedings (Cat. No.99CH36368), Victoria, BC, Canada, August 1999.

[10] X. Li, M. Amini Salehi, and M. Bayoumi, "VLSC: video live streaming using cloud services," in Proceedings of the 2016 IEEE International Conferences on Big Data and Cloud Computing (BDCloud), Social Computing and Networking (SocialCom), Sustainable Computing and Communications (SustainCom) (BDCloud-SocialCom-SustainCom), pp. 595600, IEEE, Atlanta, GA, USA, October 2016.

[11] ITU-R Recommendation BT, Methodology for the Subjective Assessment of the Quality of TelevisionPictures, ITU-R Recommendation BT, Geneva, Switzerland, 2002.

[12] N. N. Katore and N. J. Uke, "ESVP-Cloud: efficient socialaware video prefetching for adaptive multimedia streaming and video sharing in the clouds," in Proceedings of the 2015 International Conference on Applied and Theoretical 
Computing and Communication Technology (iCATccT), Davangere, India, October 2015.

[13] M. Shahid, J. Panasiuk, G. Van Wallendael, M. Barkowsky, and B. Lovstrom, "Predicting full-reference video quality measures using HEVC bitstream-based no-reference features," in Proceedings of the 2015 Seventh International Workshop on Quality of Multimedia Experience (QoMEX), pp. 1-2, IEEE, Pylos-Nestoras, Greece, May 2015.

[14] B. Appina and S. S. Channappayya, "Full-reference 3-D video quality assessment using scene component statistical dependencies," IEEE Signal Processing Letters, vol. 25, no. 6, pp. 823-827, 2018.

[15] B. Rogge, J. Bekaert, and R. Van de Walle, "Timing issues in multimedia formats: review of the principles and comparison of existing formats," IEEE Transactions on Multimedia, vol. 6, no. 6, pp. 910-924, 2004.

[16] J. Korhonen, N. Burini, J. You, and E. Nadernejad, "How to evaluate objective video quality metrics reliably," in Proceedings of the 2012 Fourth International Workshop on Quality of Multimedia Experience, pp. 57-62, IEEE, Yarra Valley, VIC, Australia, July 2012.

[17] R. Soundararajan and A. C. Bovik, "Video quality assessment by reduced reference spatio-temporal entropic differencing," IEEE Transactions on Circuits and Systems for Video Technology, vol. 23, no. 4, pp. 684-694, 2012.

[18] K. Brunnström, I. Sedano, K. Wang et al., "2D no-reference video quality model development and $3 \mathrm{D}$ video transmission quality," in Proceedings of the Sixth International Workshop on Video Processing and Quality Metrics for Consumer Electronics,(VPQM 2012), pp. 19-20, Scottsdale, AZ, USA, January 2012.

[19] O. B. Maia, H. C. Yehia, and L. D. Errico, "A concise review of the quality of experience assessment for video streaming," Computer Communications, vol. 57, pp. 1-12, 2015.

[20] Z. Wang, E. P. Simoncelli, and A. C. Bovik, "Multiscale structural similarity for image quality assessment," in Proceedings of the Conference Record of the 37th Asilomar Conference on Signals, Systems and Computers, vol. 2, pp. 1398-1402, Pacific Grove, CA, USA, November 2003.

[21] A. Pessoa, A. Falcao, R. Nishihara, A. Silva, and R. Lotufo, "Video quality assessment using objective parameters based on image segmentation," SMPTE Journal, vol. 108, no. 12, pp. 865-872, 1999.

[22] A. B. Watson, J. Hu, and J. McGowan, "Digital video quality metric based on human vision," Journal of Electronic Imaging, vol. 10, no. 1, pp. 20-29, 2001.

[23] F. Xiao, DCT-Based Video Quality Evaluation, MSU Graphics and Media Lab (Video Group), 2000, http://www. compression.ru/video/.

[24] P. Ndjiki-Nya, M. Barrado, and T. Wiegand, "Efficient fullreference assessment of image and video quality," in Proceedings of the IEEE International Conference on Image Processing (ICIP), pp. 125-128, San Antonio, TX, USA, October 2007.

[25] D. M. Chandler and S. S. Hemami, "VSNR: a wavelet-based visual signal-to-noise ratio for natural images," IEEE Transactions on Image Processing, vol. 16, no. 9, pp. 2284-2298, 2007.

[26] M. A. R. Mustafa, M. MamunElahi, M. AlamgirHossain, and M. M. Islam, "Comparative performance analysis of MPEG4, FLV and 3GP multimedia file formats using wireless network parameters," in Proceedings of the 2010 13th International Conference on Computer and Information Technology (ICCIT), pp. 306-311, IEEE, Dhaka, Bangladesh, December 2010.
[27] K. R. Laghari, O. Issa, F. Speranza, and T. H. Falk, "Qualityof-experience perception for video streaming services: preliminary subjective and objective results," in Proceedings of the Signal and Information Processing Association Summit and Conference, IEEE, Hollywood, CA, USA, December 2012.

[28] 2019, https://www.youtube.com/watch?v=aT-Dt3C1SYI.

[29] 2019, https://www.youtube.com/watch?v=_YdvWyXuJbA.

[30] 2019, https://www.youtube.com/watch?v=vrCRyqjKoO8.

[31] 2019, https://www.tubeoffline.com/download-YouTube-videos. php.

[32] http://www.compression.ru/video/quality_measure/video_ measurement_tool.html.

[33] J. L. Martínez, P. Cuenca, F. Delicado, and F. Quiles, "Objective video quality metrics: a performance analysis," in Proceedings of the 2006 14th European Signal Processing Conference, pp. 1-5, IEEE, Florence, Italy, September 2006.

[34] https://www.videolan.org/index.html. 


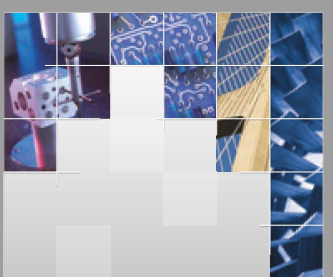

\section{Enfincering}
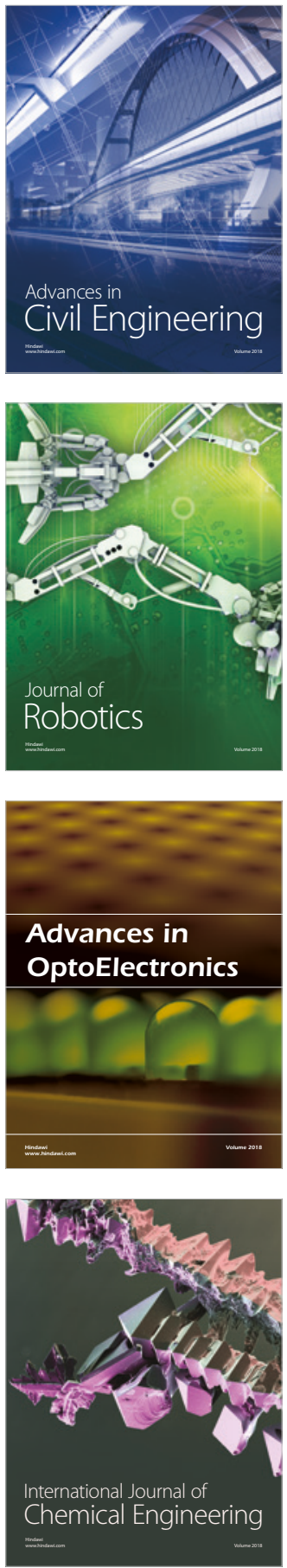

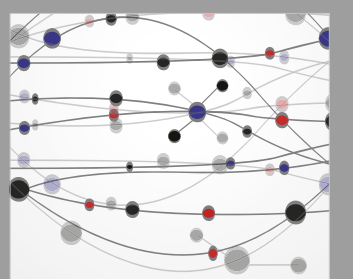

\section{Rotating \\ Machinery}

The Scientific World Journal

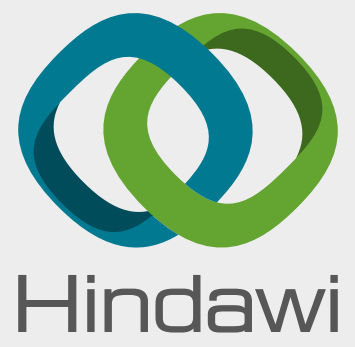

Submit your manuscripts at

www.hindawi.com
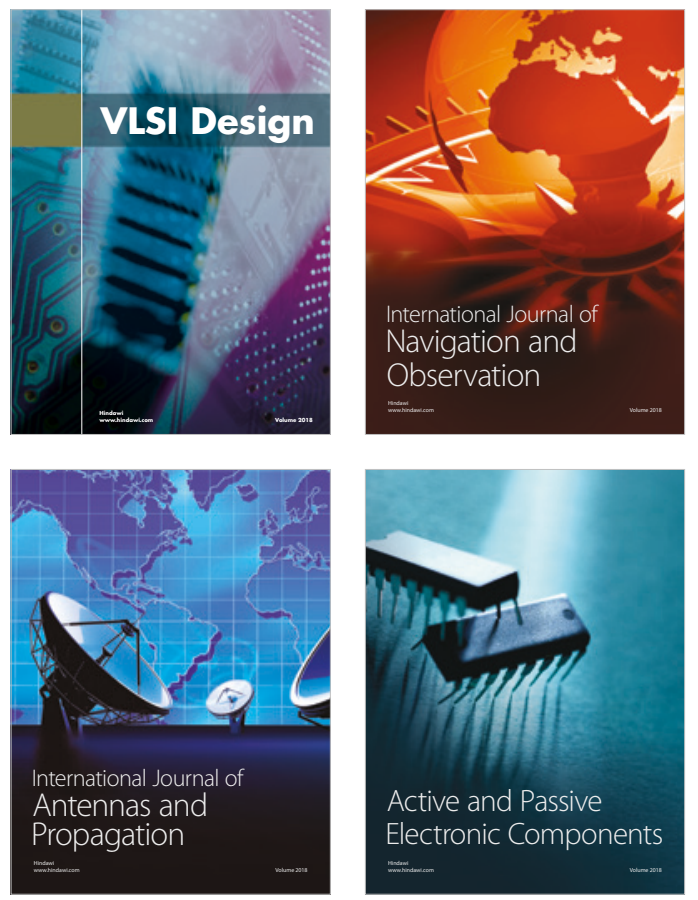
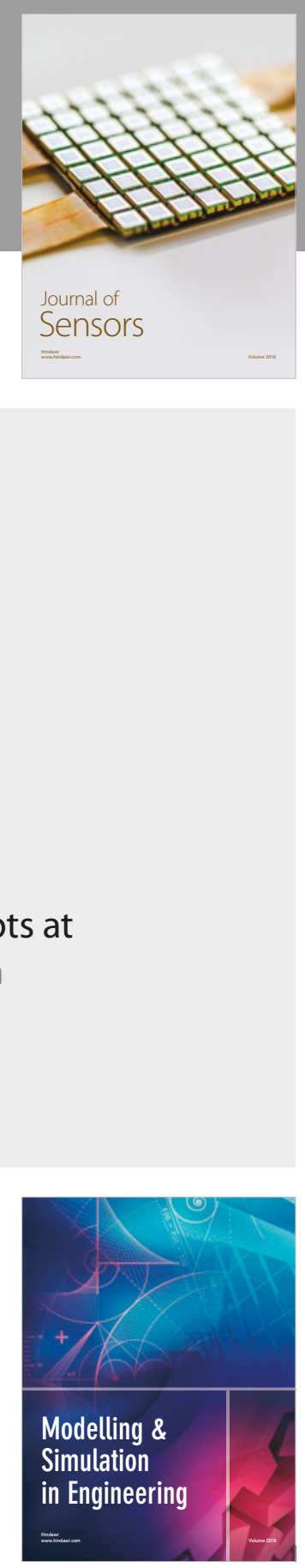

\section{Advances \\ Multimedia}
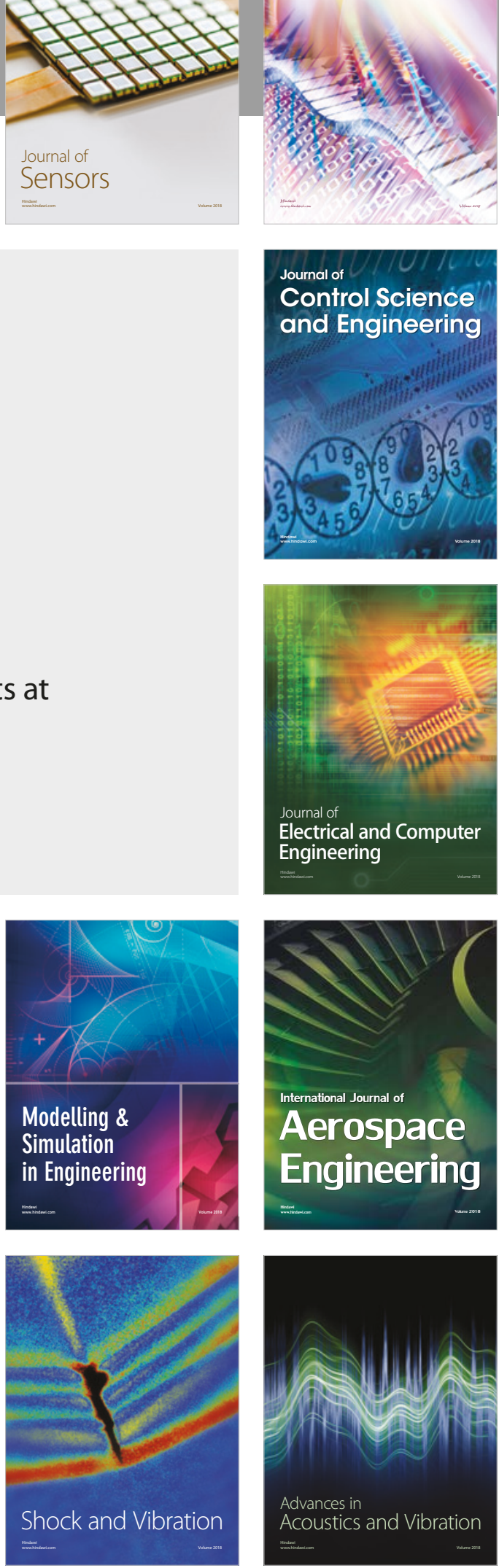